\title{
Scanning electronmicroscopy of hypertrophic and keloid scar
}

\author{
J. V. Harvey Kemble \\ M.A., F.R.C.S.
}

Wessex Regional Plastic Surgery and Burns Research Unit, Odstock Hospital, Salisbury

Summary

The scanning electronmicroscopic appearances of hypertrophic and keloid scar before and after treatment, mature scar and normal skin have been investigated. There is a graduation of features such as collagen orientation and fibre size which accompanies the clinical maturation from the active hypertrophic state into mature scar, a process which is hastened but not materially altered by treatment with steroid or compression-occlusion.

\section{Introduction}

Although the clinical features of a hypertrophic scar resulting fron injury to the dermis of the skin are well recognized, there is still much discussion as to whether such scarring is merely a more intense reaction to trauma than that which results in normal scar, or whether it is a wholly aberrant healing process. The problem is particularly acute in attempts to define keloid as distinct from or only differing in degree from hypertrophic scar.

The purpose of the investigation was to study the electronmicroscopic changes in the dermis which accompany the clinical alteration of the scar from the painful, erythematous, heaped-up hypertrophic state to the pale, flatter, mature scar, and to determine whether treatment which has resulted in clinical improvement in the scar is associated with any electronmicroscopic features which could be interpreted as indicating a totally distinct healing process.

\section{Materials and methods}

Normal skin was obtained from patients undergoing mammary or abdominal reductions. Nonhypertrophic mature scars were removed in the course of scar revisions. Keloid and hypertrophic scars were biopsied with a fine skin trephine before steroid injection therapy or compression-occlusion beneath a neoprene mould (Larson et al., 1971; Thomson, 1974), and for up to 9 months afterwards. The steroid used was triamcinolone acetonide 10 $\mathrm{mg} / \mathrm{ml}$ (Squibb) by injection directly into the scar on up to four occasions at 1-monthly intervals. In order to determine whether treatment was effective or not, part of each scar only was treated. the other part acting as control.
Samples were immediately placed in $25 \%$ gluteraldehyde in phosphate buffer and kept at $4^{\circ} \mathrm{C}$ until freeze-dried. Small cubes of sample were quench frozen in iso-pentane cooled in liquid nitrogen, and dried for $24 \mathrm{hr}$ at minimum pressure in an EdwardsPearse EPD2 unit. The freeze-dried samples were mounted on to aluminium stubs and coated with carbon followed by Au-Pd. The coated specimens were observed in a Cambridge Stereoscan Mark II instrument using $10 \mathrm{kV}$ accelerating voltage.

\section{Results}

A representative specimen of normal skin is shown in Fig. 1. There was a prominent interstitial space. The collagen fibres were diffusely distributed either into small or large bundles, and were wavy. Their orientation was approximately parallel, but this feature showed considerable variation with areas where no directional predominance was present. Thin cross-strands occurred between major collagen bundles. The capillaries were patent, and whorling was absent.

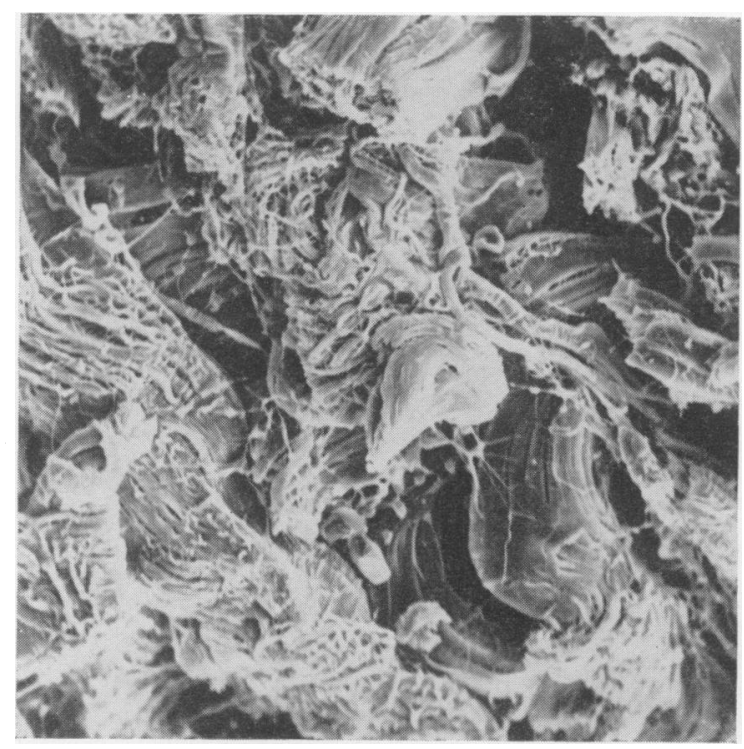

FIG. 1. Normal skin, $\times 750$. 
Non-hypertrophic mature scar contained a larger amount of collagen fibres with some bundle formation and a reduced interstitial space. 'Vacuoles' were frequent. The fibres lacked the waviness seen in normal skin, but fibre diameter was similar to that of unscarred skin (Fig. 2).

In the hypertrophic scar, the overall impression was of much increased density of collagen. The bundle arrangement was less obvious and the

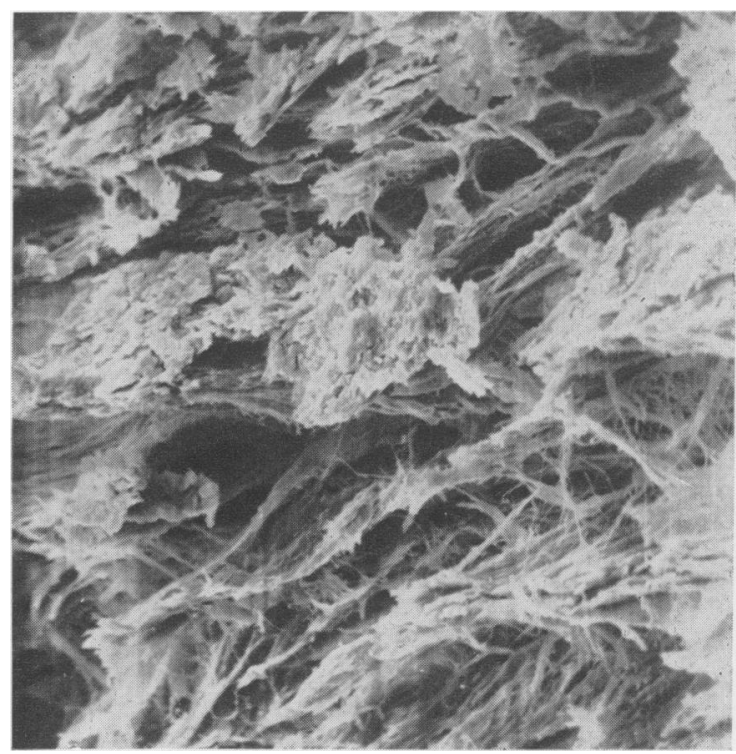

FIG. 2. Non-hypertrophic scar, $\times 750$.

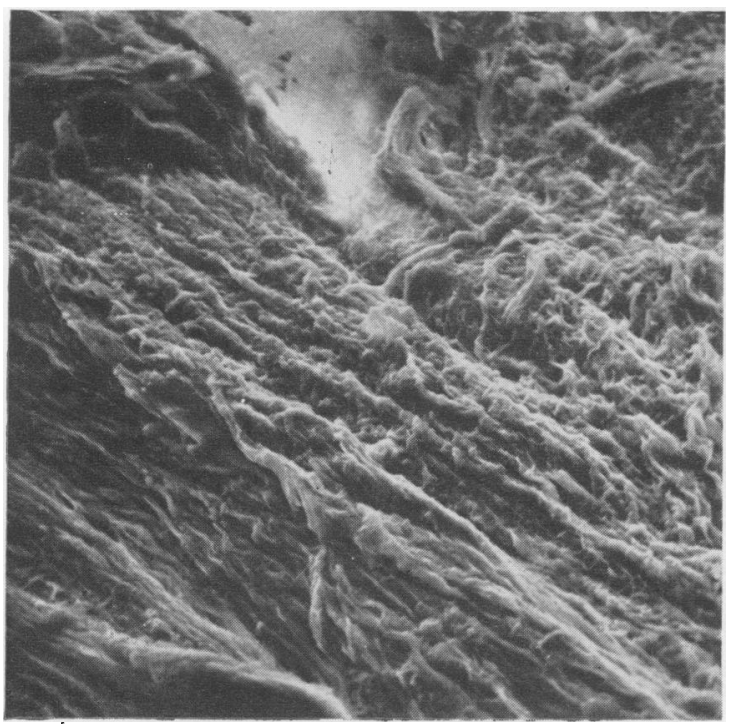

FIG. 3. Active hypertrophic scar, $\times 750$. confluence of collagen gave a homogeneous appear- $\varrho$ ance (Fig. 3). Fibre diameter was about half to three-z quarters of that in normal skin, and cross-stranding $\stackrel{\mathbb{Q}}{\varrho}$ was frequent. The fibres were for the most part $c$. straight. Capillaries were absent. Some whorled $\overrightarrow{\vec{F}}$ nodules were observed. No significant differences ? between keloid and hypertrophic scar were observed $\bar{C}$ but in both, fibre parallelism was usually marked.

The steroid-treated hypertrophic scar (Fig. 4) showed a dense homogeneous arrangement of $\cong$ collagen, with small fibre size. It differed from the active hypertrophic scar only in a relative increase in $\vec{\circ}$ interstitial space. The hypertrophic scar treated by compression-occlusion showed some parallelism of $\vec{\omega}$ small fibre bundles and some return of waviness. $\frac{\text { g }}{8}$

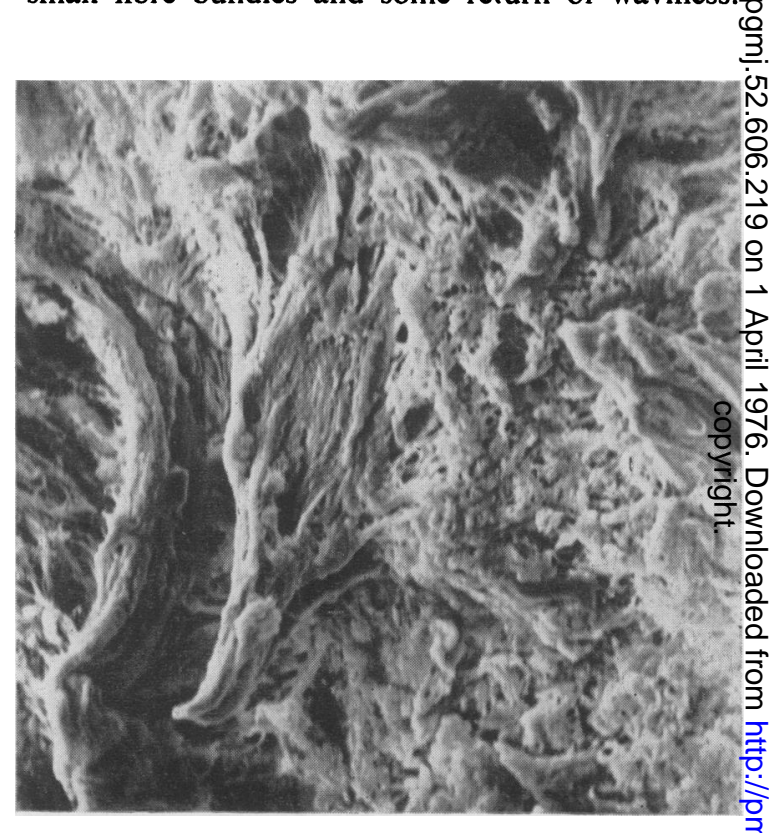

FIG. 4. Steroid treated maturing hypertrophic scar, $\times 750$.

\section{Discussion}

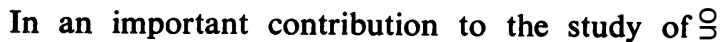
wound healing, Forrester et al. (1970) noted that in $\frac{D}{2}$ rats, moderate tension in a wound predisposed collagen fibres to align. This resulted in a higher $N$ tensile strength of the wound than that in unten. sioned scar. In clinical practice, it is well recognized 0 that release of tension in a hypertrophic scar by Z- $\mathbb{W}$ plasty or introduction of graft frequently results in a rapid maturation to a non-hypertrophic state. The $\stackrel{0}{\circ}$ author has noticed that the collagen fibres in hyper- $\bar{D}$ trophic scars when individually discernible, are often $\stackrel{\mathscr{C}}{\rightarrow}$ aligned, in addition to being more compact, and ${ }^{\circ}$ lacking the waviness characteristic of untensioned $\frac{0}{\mathbb{D}}$ scar or skin (Linares et al., 1972; Carr, 1970). 
Holmstrand, Longacre and de Stefano (1961), using an X-ray diffraction technique, found two systems of collagen fibrils at right angles in normal dermis, the better defined system running parallel to crease lines, but in the system in hypertrophic scar the collagen was orientated in the longitudinal direction of the scar. The electronmicroscopic study confirms those findings.

The reduction in ground substance and interstitial space in the hypertrophic scar results in a dense homogeneous collagen matrix in which individual bundles are considerably less distinct than in normal skin or mature scar. However as the hypertrophic scar matures clinically, the arrangement more closely approximates that of normal skin, and fibre diameter increases. Appreciable loss of individual fibre recognition, parallelism, homogeneity, small fibre size, reduction of interstitial space and the presence of whorls are features of the hypertrophic scar. The arrangement of fibres into bundles, the less obvious parallel orientation, the appearance of 'vacuoles' and enlarged interstitial space, larger fibre size approximating to that of normal dermis and the absence of whorls are characteristic of mature scar.

The appearances of the steroid-treated and compression-occlusion treated scars are intermediate between active hypertrophic scar and mature scar. There are no recognizable features which can be attributed specifically to treatment. It is therefore probable that any effect produced by treatment is merely a hastening of the normal processes of scar maturation, and that hypertrophic and keloid scars differ only in degree of reaction, rather than in any fundamental way, from normal scar.

Histological and histochemical studies have shown that steroids affect fibroblasts, collagen and interstitial mucopolysaccharides (Dougherty and Schneebeli, 1955; Berliner, Panagiotis and Nabors, 1967). Berliner and Ruhmann (1967) demonstrated a decreased growth of fibroblasts in culture after introduction of fluocinolone. There exists in the extrafibrillar compartment of skin a collagenolytic zymogen which releases hydroxyproline from in- soluble collagen. In addition, following cortisol injection, an extracellular extrafibrillar protease attacks ground substance proteins to which acid mucopolysaccharides are bound (Houck, 1964). The electronmicroscopic appearances of hypertrophic scar show little extrafibrillar extracellular space, but significantly more in the maturing steroid-treated scar. This is probably a reflection of the diminished fibroblast production of collagen, as well as an increase in collagen lysis.

\section{Acknowledgments}

The author wishes to thank most sincerely Mr Mark Ainsworth, Chemical Defence Establishment, Porton, for access to the electronmicroscope, and Mrs Daphne Graiff and Mr Roger Brown for their technical expertise.

\section{References}

Berliner, D.L. \& RuhmanN, A.G. (1967) Influence of steroids on fibroblasts. Journal of Investigative Dermatology, 49, 117.

Berliner, D.L., Panagiotis, N.M. \& Nabors, C.J. (1967) Ultrastructure of fibroblasts following topical corticosteroid treatment of wounds. Endocrinology, 81, 461.

CARR, K.E. (1970) Scanning electronmicroscope studies in human skin. British Journal of Plastic Surgery, 23, 66.

Dougherty, T.F. \& SChNeEbeli, G.L. (1955) Use of steroids as anti-inflammatory agents. Annals of New York Academy of Sciences, 61, 328.

Forrester, J.C., Zederfeldt, B.H., Hayes, T.L. \& Hunt, T.K. (1970) Tape closed and sutured wounds: a comparison by tensiometry and scanning electronmicroscopy. British Journal of Surgery, 57, 729.

Holmstrand, K., LongaCRe, J.J. \& De STEFano, G.A. (1961) Ultrastructure of collagen in skin, scars and keloids. Plastic and Reconstructive Surgery, 27, 597.

Houck, J.C. (1964) Mode of action of cortisol on the connective tissue. Arthritis and Rheumatism, 7, 738.

Larson, D.L., Abston, S., Evans, E.B., Dobrkovsky, M. \& LinARES, H.A. (1971) Techniques for decreasing scar formation in the burned patient. Journal of Trauma, 11, 807.

LinARES, H.A., KISCHER, C.W., DOBRKovsKy, M. \& LARSON, D.L. (1972) The histiotypic organization of the hypertrophic scar in humans. Journal of Investigative Dermatology, 59, 323.

Thomson, W.G. (1974) Treatment of hypertrophic scarring by compression and occlusion. Proceedings of the Royal Society of Medicine, 67, 256. 